\title{
Prokaryotically and eukaryotically expressed interleukin-24 induces breast cancer growth suppression via activation of apoptosis and inhibition of tumor angiogenesis
}

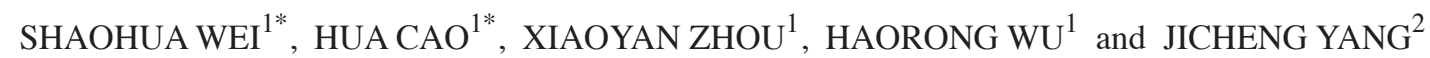 \\ ${ }^{1}$ Department of General Surgery, The Second Affiliated Hospital of Soochow University, Suzhou, Jiangsu 215004; \\ ${ }^{2}$ Department of Cell and Molecular Biology, College of Medicine, Soochow University, Suzhou, Jiangsu 215123, P.R. China
}

Received December 25, 2013; Accepted October 24, 2014

DOI: $10.3892 / \mathrm{mmr} .2014 .3136$

\begin{abstract}
Melanoma differentiation-associated-7 (mda-7)/interleukin-24 (IL-24), a unique cytokine-tumor suppressor, exerts tumor-selective killing activity in numerous types of cancer cell. Although eukaryotically and prokaryotically expressed recombinant human (rh)IL-24 proteins have been previously shown to produce potent antitumor effects, to the best of our knowledge, no side-by-side study has been conducted that compares the two proteins directly. In the present study, rhIL-24 protein was expressed in BL21 Escherichia coli transformed with the pET-21a(+)-hIL-24 plasmid by isopropyl- $\beta$-D-1-thiogalactopyra noside induction. Following a denaturing and renaturing process, the soluble rhIL-24 was purified using a Q-Sepharose column. rhIL-24 protein was also expressed in Chinese hamster ovary mammalian cells stably transfected with the pcDNA3-hIL-24 plasmid. The in vitro antitumor efficacies of the two treatments were compared using the MDA-MB-231 human breast cancer cell line. Furthermore, the therapeutic efficacies of the bacteria-derived rhIL-24 protein and the liposome-coated pcDNA3-hIL-24 naked plasmid were evaluated in athymic nude mice with subcutaneously xenografted MDA-MB-231 cell tumors. The prokaryotically expressed/purified rhIL-24 protein and the eukaryotically expressed rhIL-24 in the cell
\end{abstract}

Correspondence to: Dr Shaohua Wei, Department of General Surgery, The Second Affiliated Hospital of Soochow University, 1055 Sanxiang Road, Suzhou, Jiangsu 215004, P.R. China

E-mail: shaohuaw321@163.com

Professor Jicheng Yang, Department of Cell and Molecular Biology, College of Medicine, Soochow University, 199 Renai Road, Suzhou, Jiangsu 215123, P.R. China

E-mail: jcyang@suda.edu.cn

${ }^{*}$ Contributed equally

Key words: melanoma differentiation-associated-7/interleukin-24, prokaryotic expression, eukaryotic expression, breast cancer, antitumor activity supernate were revealed to be capable of efficiently suppressing MDA-MB-231 tumor growth in vitro. Similarly, the administration of bacteria-derived rhIL-24 protein and pcDNA3-hIL-24 naked plasmid also provided therapeutic benefits in the treatment of in vivo MDA-MB-231 xenografted tumors. The retarded in vitro and in vivo breast cancer growth elicited by rhIL-24 was closely associated with the upregulation of the ratio of anti-apoptotic B cell lymphoma 2 (Bcl-2) to pro-apoptotic $\mathrm{Bcl}-2$-associated $\mathrm{X}$ protein (Bax), as well as the activation of caspase-3 followed by marked induction of apoptosis, and the notable inhibition of tumor angiogenesis. Thus, the results of the present study indicate that prokaryotically expressed rhIL-24 protein may be an alternate and promising antitumor agent in human breast cancer or other types of cancer.

\section{Introduction}

Melanoma differentiation-associated-7 (mda-7)/interleukin-24 (IL-24), a member of the IL-10 family of cytokines, is a unique tumor suppressor (1). A number of studies have demonstrated that IL-24 exhibits broad spectrum antitumor activity without damaging normal cells $(2,3)$. Forced IL-24 expression and exposure to IL-24 induces cell-cycle alteration, apoptosis and toxic autophagy, and consequently elicits growth inhibition in numerous types of cancer cell $(2,3)$. IL-24 also directly inhibits vascular endothelial cell differentiation and migration via the IL-20R2/IL-22R heterodimeric receptor (4) and indirectly represses the production of the vascular endothelial growth factor and IL-8 proangiogenic factors $(5,6)$, resulting in the suppression of tumor angiogenesis. In addition, IL-24 exerts marked immunomodulatory activity and enhances antitumor immunity through the induction of robust production of IL-6, tumor necrosis factor- $\alpha$ and interferon- $\gamma$ (7). Furthermore, IL-24 efficiently impairs tumor invasion and migration through the downregulation of phosphatidylinositide 3-kinase (PI3K), focal adhesion kinase and matrix metalloproteinases (8). Notably, IL-24 exerts potent antitumor bystander activity via IL-20R1/IL-20R2 and IL-20R2/IL-22R-mediated autocrine/paracrine signaling (2,9). Most importantly, IL-24 sensitizes cancer cells to radiation-, chemotherapy-, monoclonal antibody- and histone deacetylase inhibitor-induced antitumor effects $(2,3,10)$. Thus, IL-24 is a multifunctional 
tumor suppressing cytokine and is currently regarded as a 'magic bullet' for cancer.

Breast cancer is the most common type of malignancy (23\%) among females worldwide and is the second leading cause of cancer-associated mortality in females (14\%) (11). Conventional therapeutic methods for breast cancer include surgery, chemotherapy, radiation therapy, hormone therapy and targeted therapy, or a combination of these treatments (12). In spite of significant advances in early detection and considerable clinical therapeutic success, de novo and acquired resistance currently remains a therapeutic challenge in breast cancer treatment. Although the primary lesion may be eradicated, the disease is resistant to the majority of conventional therapeutic methods, which frequently results in relapse and metastasis, thus warranting the search for novel therapeutic strategies and agents for use in breast cancer therapy.

IL-24 has been shown to be substantially downregulated in breast cancer, which contributes to adverse pathological features and poor clinical outcomes $(13,14)$. A preclinical animal study revealed that adenovirus-mediated IL-24 gene therapy (Ad.mda-7) is a promising candidate for the treatment of breast cancer (15). The bacteria fusion protein glutathione-S-transferase-IL-24, a novel therapeutic agent, also exerts cancer-selective killing activity (16). Furthermore, bacteria-derived arginine-glycine-aspartic acid-modified IL-24 protein exhibits enhanced antitumor activity against breast cancer via targeting the $\alpha \mathrm{V} \beta 3$ and $\alpha \mathrm{V} \beta 5$ integrin receptors (17). However, to the best of our knowledge, no side-by-side studies that compare the antitumor effects of eukaryotically and bacterially expressed recombinant human IL-24 (rhIL-24) protein have been conducted. In the present study, the rhIL-24 protein was expressed in BL21 bacteria transformed with the pET-21a(+)-hIL-24 plasmid and eukaryotic Chinese hamster ovary $(\mathrm{CHO})$ cells stably transfected with the pcDNA3-hIL-24 plasmid, and the antitumor efficacy and underlying mechanisms of the two treatments were directly compared in vitro using the MDA-MB-231 human triple-negative breast cancer cell line that expresses the IL-20R1/IL-20R2 receptor (17). The in vivo therapeutic efficacy of the bacterial rhIL-24 protein and the pcDNA3-hIL-24 naked plasmid were also analyzed using subcutaneously (s.c.) xenografted MDA-MB-231 breast cancer cell tumors.

\section{Materials and methods}

Plasmids, cell lines, reagents and mice. The pUC19-hIL-24 cloning vector containing full-length human IL-24 cDNA was constructed in the laboratory of the Department of Cell and Molecular Biology, Soochow University (Suzhou, China). The pET21a(+) prokaryotic expression plasmid and BL21 bacteria were purchased from Novagen (Beijing, China). The pcDNA3 eukaryotic expression plasmid, Lipofectamine ${ }^{\mathrm{TM}} 2000$ and neomycin (G418) were obtained from Invitrogen (Shanghai, China). The MDA-MB-231 human breast cancer cell line and the $\mathrm{CHO}$ cell line were bought from the American Type Culture Collection (Rockville, MD, USA). The QBI-293A human embryonic kidney cell line was provided by Professor Jiang Zhong (Department of Microbiology, College of Life Science, Fudan University, Shanghai, China). The MDA-MB-231, QBI-293A and CHO cells were cultured in
RPMI-1640 medium (Invitrogen) supplemented with $10 \%$ fetal bovine serum (HyClone, Logan, UT, USA). AxyPrep Plasmid Miniprep, AxyPrep DNA Gel Extraction and AxyPrep DNA Purification kits were purchased from Axygen Biosciences (Union City, CA, USA). BamHI, XhoI, KpnI and XbaI restriction endonucleases, T4 DNA ligase, DL2000 DNA marker and isopropyl- $\beta$-D-1-thiogalactopyranoside (IPTG) were obtained from Takara Biotechnology Co., Ltd. (Dalian, China). The 3-(4,5-dimethylthiazol-2-yl)-2,5-diphenyltetrazolium bromide (MTT)kit and the mammalian cell lysis kit were purchased from Sigma-Aldrich (Shanghai, China). The Annexin V-fluorescein isothiocyanate (FITC)/propidium iodide (PI) apoptosis detection kit was provided by BD Biosciences (Shanghai, China). The IL-24 antibody and the IL-24 enzyme-lined immunosorbent assay (ELISA) kit were obtained from R\&D Systems (Shanghai, China). The antibodies specific for B cell lymphoma 2 (Bcl-2), Bcl-2-associated X protein (Bax), cleaved caspase-3, cluster of differentiation (CD) 34 and $\beta$-actin were purchased from Cell Signaling Technology, Inc. (Boston, MA, USA). The terminal deoxynucleotidyl transferase-mediated dUTP nick end labeling (TUNEL) apoptosis detection kit was bought from Beyotime Institute of Biotechnology (Beijing, China). The UltraSensitive ${ }^{\mathrm{TM}}$ SP kit was provided by Fuzhou Maixin Biotechnology Development Co., Ltd. (Fuzhou, China). Four-week-old male athymic BALB/c nude mice were purchased from Shanghai Experimental Animal Center (Shanghai, China) and were maintained in the animal facility at Soochow University (Suzhou, China) according to the Soochow University animal research committee guidelines. The present study was approved by the ethics committee of Soochow University.

Construction of recombinant vectors expressing human IL-24. The cDNA fragment encoding full-length mature human IL-24 protein minus the signal peptide was amplified by polymerase chain reaction using the pUC19-hIL-24 cloning plasmid as a template and the following pair of primers specific for mature human IL-24: Forward: 5'-CGGATCCATGCAGGG CCAAGAATTCCAC-3' and reverse: 5'-CCTCGAGCTAGA GCTTGTAGAATTTCT-3'. The primers were subsequently subcloned into the pET-21a(+)prokaryotic expression plasmid at the BamHI and XhoI sites to generate pET-21a(+)-hIL-24. The cDNA fragment encoding the full-length human IL-24 protein containing the signal peptide was directly released from the pUC19-hIL-24 plasmid using KpnI and XbaI double digestion, and was subsequently subcloned into the pcDNA3 eukaryotic expression plasmid at the KpnI and $\mathrm{XbaI}$ sites to form pcDNA3-hIL-24.

Expression of rhIL-24. To analyze human IL-24 expression in a prokaryotic system, BL21 Escherichia coli (E. coli) was transformed with either the pET-21a(+)-hIL-24 construct or the pET-21a(+) control plasmid, and the bacteria were treated with IPTG according to the IPTG kit manufacturer's instructions. Briefly, the BL21/pET-21a(+)-hIL-24 and BL21/pET-21a(+) plasmids were incubated overnight in Luria-Bertani (LB) medium (Sangon Biotech Co., Ltd, Shanghai, China) containing $100 \mu \mathrm{g} / \mathrm{ml}$ ampicillin (Amp; Sangon Biotech Co., Ltd). Subsequently, the bacteria were transferred at a $2 \%$ dilution into fresh LB medium with 
$100 \mu \mathrm{g} / \mathrm{ml}$ Amp. When optical density at $600 \mathrm{~nm}$ reached $\sim 0.6$, as measured using a SmartSpec 3000 spectrophotometer (Bio-Rad Laboratories, Inc., Hercules, CA, USA), the bacteria were induced using IPTG at a final concentration of $1 \mathrm{mM}$ and then harvested at different time points $(0-5 \mathrm{~h})$. To examine human IL-24 expression in a eukaryotic system, the QBI-293A and CHO mammalian cells were transfected with either the pcDNA3-hIL-24 plasmid or the pcDNA3 control plasmid using Lipofectamine 2000 according to the manufacturer's instructions. After $72 \mathrm{~h}$ transfection, the supernates and the cell lysates from the QBI-293A transfectants (a high-efficiency transfection cell line) were collected for the measurement of human IL-24 expression levels. At $48 \mathrm{~h}$ after transfection, the $\mathrm{CHO}$ transfectants were cultured with $1 \mathrm{mg} / \mathrm{ml}$ neomycin for 2-3 weeks, replacing culture medium every 3-4 days. CHO/pcDNA3-hIL-24-positive clones expressing IL-24 were further selected and amplified.

Purification of bacterial rhIL-24 protein. Following the establishment of optimal conditions for prokaryotic expression, the rhIL-24 protein was abundantly induced at the $3 \mathrm{~h}$ time point following $1 \mathrm{mM}$ IPTG treatment of BL21/pET-21a(+)-hIL-24 bacteria. The bacteria were harvested at 1,500 $\mathrm{x} g$ and washed with $0.9 \% \mathrm{NaCl}$ solution. Subsequently, the samples were sonicated (Shunmayq, Inc., Nanjing, China) at a high setting (duty time, $30 \mathrm{sec}$; rest time, $30 \mathrm{sec}$ ) at $480 \mathrm{~W}$ in an ice bath and centrifuged at $1,500 \mathrm{x} \mathrm{g}$ at $4^{\circ} \mathrm{C}$, and the pellets were resuspended in washing buffer (Sangon Biotech Co., Ltd) containing 20\% glycerol (v/v), 2\% Triton X-100 (v/v), $2 \mathrm{M}$ urea, $50 \mathrm{mM} \mathrm{NaCl}, 50 \mathrm{mM}$ ethylenediaminetetraacetic acid (EDTA) and $100 \mathrm{mM}$ Tris- $\mathrm{HCl}$ (pH 8.0). Following three cycles of washing, the deposit was denatured in $7 \mathrm{M}$ urea with $5 \mathrm{mM}$ dithiothreitol for $4 \mathrm{~h}$. The supernate was collected by centrifugation at $3,600 \mathrm{x}$ at $4^{\circ} \mathrm{C}$, and was placed in renaturation solution containing $2 \mathrm{M}$ urea, $100 \mu \mathrm{M}$ PMSF, $50 \mathrm{mM}$ EDTA and $100 \mathrm{mM}$ Tris- $\mathrm{HCl}\left(\mathrm{pH} \mathrm{8.2)}\right.$ at $4^{\circ} \mathrm{C}$ for $24 \mathrm{~h}$ with constant agitation. Subsequently, the renaturation solution containing the rhIL-24 protein was dialyzed for $24 \mathrm{~h}$ using phosphate-buffered saline (PBS; Sangon Biotech Co., Ltd), replacing the PBS every $4 \mathrm{~h}$. The resultant solution containing the renatured rhIL-24 protein was loaded onto a pre-equilibrated Q-Sepharose column (GE Healthcare, Beijing, China) according to the manufacturer's instructions. Following a further equilibration step, the renatured rhIL-24 protein was eluted and collected.

SDS-PAGE and western blot analysis. The IPTG-induced $(1-5 \mathrm{~h})$ and uninduced $(0 \mathrm{~h})$ BL21/pET-21a(+)-hIL-24 or BL21/pET-21a(+), and the purified rhIL-24 protein samples were boiled in SDS sample loading buffer with $\beta$-methasone (Sangon Biotech Co., Ltd) and resolved by $12 \%$ SDS-PAGE, respectively. The prokaryotic rhIL-24 protein present in the gels was detected with Coomassie brilliant blue R-250 staining. The IPTG-induced BL21/pET-21a(+)-hIL-24 and purified rhIL-24 protein were then transferred to a nitrocellulose membrane (Sangon Biotech Co., Ltd). The membrane was immunoblotted with mouse anti-human IL-24 monoclonal antibody (1:1,000; Clone 244202, Cat. MAB1965; $\mathrm{R} \& \mathrm{D}$ Systems) for $1 \mathrm{~h}$ at $37^{\circ} \mathrm{C}$ and washed with Tris-buffered saline with Tween 20 (Sangon Biotech Co., Ltd) three times, followed by incubation with horseradish peroxidise-labeled goat anti-mouse immunoglobulin G (1:3,000; sc-2005; Santa Cruz Biotechnology, Inc., Dallas, Texas, USA) for another $1 \mathrm{~h}$ at $37^{\circ} \mathrm{C}$. Subsequently, the membranes were washed and developed using a SuperEnhanced chemiluminescence detection kit (Applygen Technologies Inc., Beijing, China) following the manufacturer's instructions. The protein bands were visualized following exposure of the membrane to Kodak X-ray film (Kodak, Rochester, NY, USA).

ELISA. The culture supernates derived from the pcDNA3-hIL-24- and pcDNA3-transfected QBI-293A cells, and the $\mathrm{CHO}$ cells were collected, and the quantities of rhIL-24 in the supernates were assessed using the human IL-24 ELISA kit according to the manufacturer's instructions.

MTT assay. The in vitro cytotoxicity of rhIL-24 to MDA-MB-231 human breast cancer cells was assessed using an MTT assay. Briefly, the MDA-MB-231 tumor cells ( $1 \times 10^{4}$ cells/well) were dispensed in a 96-well culture plate. At $24 \mathrm{~h}$ after culture, the tumor cells were treated with the purified prokaryotic rhIL-24 protein at a final concentration of $5 \mathrm{ng} / \mathrm{ml}$ for the indicated time periods (0-5 days). The medium containing PBS without rhIL-24 served as a control. Another group of tumor cells were incubated with either medium containing $50 \%(\mathrm{v} / \mathrm{v})$ of the supernate with IL-24 derived from the $\mathrm{CHO} / \mathrm{pcDNA} 3-\mathrm{hIL}-24$ stable transgenic cells (IL-24 final concentration, $5 \mathrm{ng} / \mathrm{ml}$ ) or the control supernate from $\mathrm{CHO} / \mathrm{pcDNA} 3$ cells. Prior to treatment and at different time points following treatment, the viability of the MDA-MB-231 tumor cells was determined by an MTT assay according to the manufacturer's instructions.

Flow cytometric analysis. The in vitro effect of rhIL-24 treatment on the apoptosis of MDA-MB-231 human breast cancer cells was evaluated by flow cytometry. A total of $1 \times 10^{6}$ MDA-MB-231 tumor cells were treated with purified prokaryotic rhIL-24 protein $(5 \mathrm{ng} / \mathrm{ml})$ or $\mathrm{PBS}$, and $50 \%(\mathrm{v} / \mathrm{v})$ of the supernate with IL-24 (IL-24 final concentration, $5 \mathrm{ng} / \mathrm{ml}$ ) or control supernate, respectively. At 24-72 h after treatment, the MDA-MB-231 tumor cells were collected and washed in cold PBS. Apoptosis was analyzed by flow cytometry using Annexin V-FITC (early apoptotic marker) and PI (late apoptotic marker) double-staining following the manufacturer's instructions. Briefly, $1 \times 10^{5}$ tumor cells were incubated with $5 \mu \mathrm{l}$ Annexin V-PE and $5 \mu 1$ 7-amino-actinomycin D in $100 \mu 11 \mathrm{X}$ Annexin $\mathrm{V}$ binding buffer at room temperature. After $15 \mathrm{~min}$ incubation, $400 \mu 11 \mathrm{X}$ binding buffer was added and the numbers of apoptotic cells were determined by flow cytometry.

Animal experiments. The male athymic BALB/c nude mice were s.c. inoculated in the armpits of the right anterior limbs with $1 \times 10^{6}$ MDA-MB-231 human breast cancer cells and were then monitored daily for tumor growth. Tumor volume was measured with a caliper and was calculated by the following formula: Tumor size $=a b^{2} / 2$, in which $\mathrm{a}$ is the larger and $\mathrm{b}$ is the smaller of the two dimensions. When the MDA-MB-231 s.c. xenografted tumors had reached a mean tumor volume of $\sim 100 \mathrm{~mm}^{3}$, the tumor-bearing mice were then subjected to in vivo treatment experiments. The tumor-bearing mice were randomly divided 
A

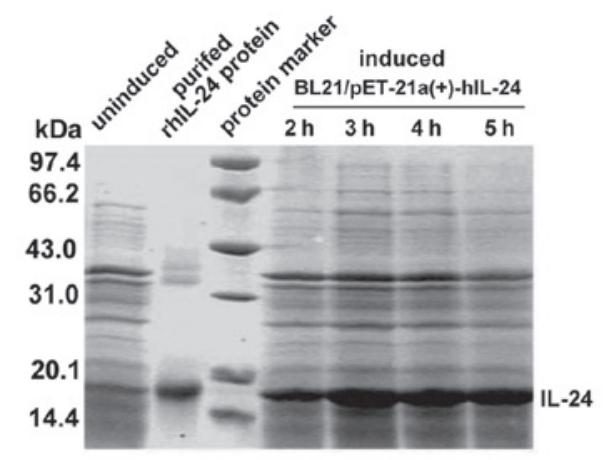

B

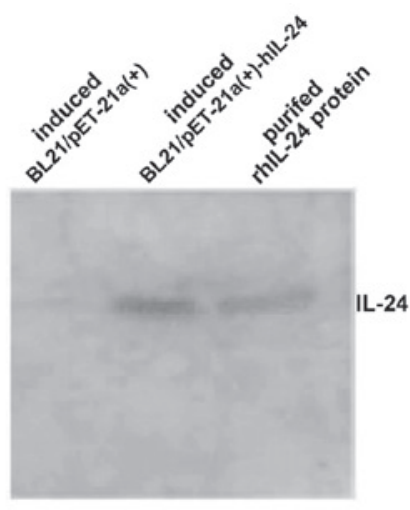

C

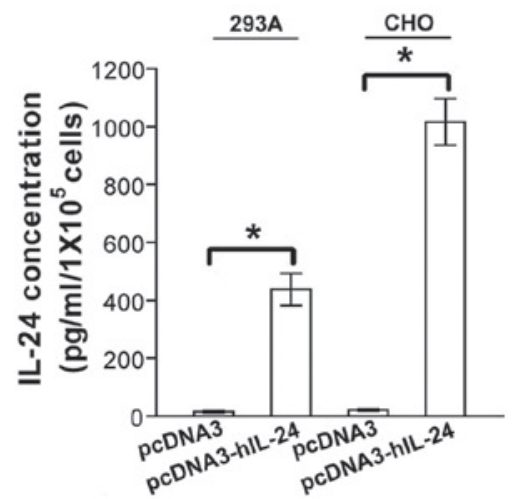

D
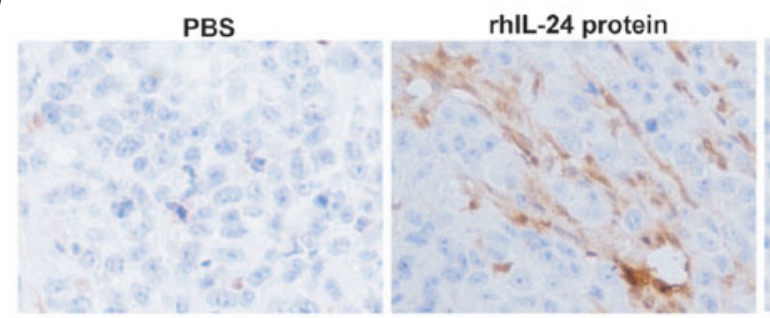

pcDNA3

pcDNA3-hIL-24

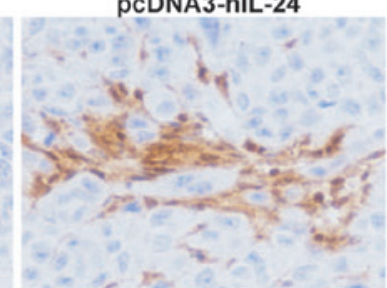

Figure 1. Recombinant expression of human interleukin-24 (IL-24) in vitro and in vivo. (A) SDS-PAGE analysis of recombinant human IL-24 (rhIL-24) protein derived from a prokaryotic system. The uninduced and induced (2-5 h) Escherichia coli BL21/pET-21a(+)-hIL-24 strain and the purified rhIL-24 protein were resolved in 12\% SDS-PAGE and detected with Coomassie brilliant blue R-250 staining. (B) Western blot analysis of rhIL-24 protein derived from a prokaryotic system. The induced ( $3 \mathrm{~h})$ BL21/pET-21a(+)-hIL-24 and BL21/pET-21a(+) (blank vector control), and the purified rhIL-24 protein were immunoblotted with anti-human IL-24 primary antibody followed by horseradish peroxidase-labeled secondary antibody for enhanced chemiluminescence analysis. A representative image of the western blot analysis is shown. (C) ELISA analysis of rhIL-24 in the supernate derived from a eukaryotic system. The supernate from pcDNA3-hIL-24 transiently transfected QBI-293A mammalian cells and pcDNA3-hIL-24 stably transfected CHO mammalian cells was harvested, and the levels of rhIL-24 secretion were measured by ELISA. ${ }^{*} \mathrm{P}<0.05$, as compared with the control. Student's t-test, $\mathrm{n}=3$ replicates per condition, $\mathrm{n}=4$ test replicates per replicate. (D) Immunohistochemical analysis of human IL-24 expression in vivo. Representative images of immunohistochemical detection of IL-24 in MDA-MB-231 subcutaneously xenografted tumors are shown. The data are representative of three independent experiments. rhIL-24, recombinant human interleukin-24; CHO, Chinese hamster ovary; PBS, phosphate-buffered saline.

into four groups (eight mice in each group) and intratumorally injected with $2 \mu \mathrm{g}$ prokaryotic rhIL-24 protein or PBS (serving as a control), and a 1:1 (v/v) mixture of Lipofectamine 2000, and $10 \mu \mathrm{g}$ pcDNA3-hIL-24 or $10 \mu \mathrm{g}$ pcDNA3 (control) every other day a total of six times. Tumor progression and regression were monitored daily. The tumor-bearing mice were sacrificed three weeks after treatment. The xenografted tumors were removed, weighted, fixed by $10 \%$ neutral formalin and embedded in paraffin for hematoxylin and eosin staining, and immunohistochemical analysis.

Immunohistochemical analysis. The in vivo expression levels of Bcl-2, Bax, cleaved caspase-3, CD34 and IL-24 in the treated and untreated s.c. xenografted MDA-MB-231 human breast cancer cell tumor sections were examined by immunohistochemistry using the UltraSensitive ${ }^{\mathrm{TM}}$ SP kit according to the manufacturer's instructions. The detection of buffy or brown diaminobenzidine precipitates is indicative of positive reactivity. The microvessel density (MVD) in the tumor sections was examined by CD34 immunostaining, as previously described (18). Any endothelial cell cluster immunoreactive for CD34 clearly separated from the adjacent microvessels was considered a single countable vessel. To analyze the numbers of apoptotic cells in the MDA-MB-231 xenografted tumors, the tumor sections were further examined for the presence of apoptosis using the TUNEL apoptosis detection kit according to the manufacturer's instructions. Each value indicates the number of immunoreactive cells, microvessels or apoptotic cells counted at a high-power microscopic view (x200). The mean value signifies the average number derived from five high-power fields from each sample.

Statistical analysis. All data are presented as the mean \pm standard deviation. The significance of the difference between groups was evaluated by Student's t-test with SPSS 10.0 software (SPSS, Inc., Chicago, IL, USA). P $<0.05$ was considered to indicate a statistically significant difference.

\section{Results}

Expression and purification of rhIL-24. Following IPTG induction, the $E$. coli $\mathrm{BL} 21 / \mathrm{pET}-21 \mathrm{a}(+)-\mathrm{hIL}-24$ strain expressed a protein band at $\sim 18.5 \mathrm{kDa}$ molecular weight, which was particularly evident after $3 \mathrm{~h}$ induction (Fig. 1A). This is consistent with the theoretical molecular weight of unmodified mature human IL-24 protein. This band did not appear in the IPTG-uninduced strain or the IPTG-induced BL21/pET-21a(+) control strain (data not shown). Western blot analysis (Fig. 1B) further verified that this band was the human IL-24 protein. To purify the rhIL-24 protein derived from the prokarytotic expression system, the BL21/pET-21a(+)-hIL-24 stain was abundantly 
A

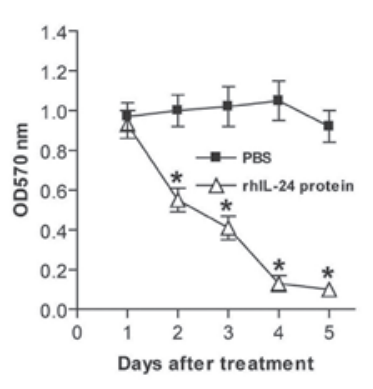

B

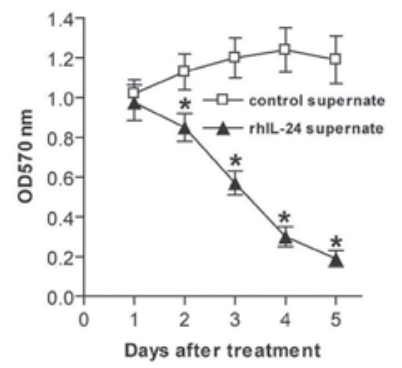

C

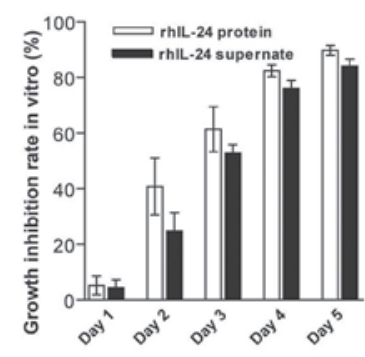

D

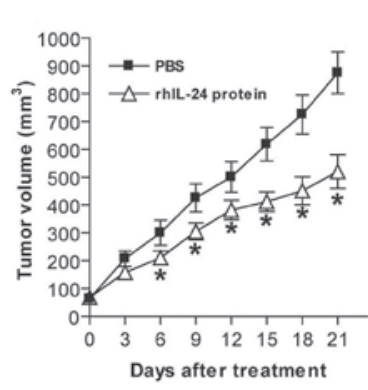

$\mathbf{E}$

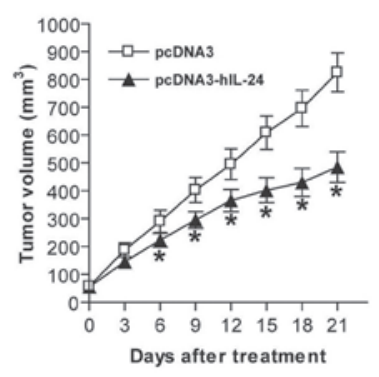

F

G
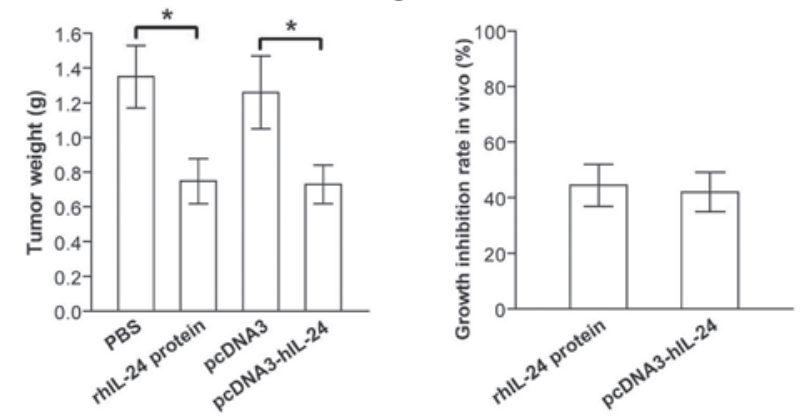

Figure 2. rhIL-24 inhibits tumor cell growth in vitro and in vivo. (A) In vitro cytotoxicity of purified bacteria rhIL-24 protein. MDA-MB-231 human breast cancer cells were treated with rhIL-24 protein. Cell survival was evaluated by MTT assay at days $1-5$ after treatment ${ }^{*} \mathrm{P}<0.05$ compared with the PBS control treatment. Data were analyzed by the Student's t-test; $n=4$ replicates per condition. (B) In vitro cytotoxicity of rhIL-24 supernate. The MDA-MB-231 tumor cells were treated with $50 \%$ (v/v) rhIL-24 supernate. Cell survival was evaluated by an MTT assay at days $1-5$ after treatment. * P<0.05 compared with the control supernate treatment. Data were analyzed by the Student's t-test; $\mathrm{n}=4$ replicates per condition. (C) In vitro growth inhibition rate. The in vitro tumor growth inhibition rate was calculated by the absorbance values [optical density (OD), $570 \mathrm{~nm}$ ] compared with those of PBS (white column) or control supernate (black column) untreated control groups. (D-G) rhIL-24 suppresses tumor cell growth in vivo. Athymic BALB/c nude mice bearing subcutaneously xenografted MDA-MB-231 cell tumors were intratumorally injected with rhIL-24 protein ( $\mu \mathrm{g}$ ) or phosphate-buffered saline (PBS), and a 1:1 (v/v) mixture of Lipofectamine ${ }^{\circledR} 2000$ and pcDNA3-hIL-24 (10 $\left.\mu \mathrm{g}\right)$ or pcDNA3 $(10 \mu \mathrm{g})$ every other day for a total of six times, respectively. (D and E) The MDA-MB-231 xenografted tumor volumes prior and subsequent to treatment were measured. (F) The xenografted tumors were removed three weeks after treatment and then weighted. ${ }^{*} \mathrm{P}<0.05$ compared with the respective control conditions. Data were analyzed by the Student's $\mathrm{t}$-test; $\mathrm{n}=8 \mathrm{replicates}$ per condition. (G) The in vivo tumor growth inhibition rate was calculated by comparing the tumors treated with the two variants of IL-24 with those of the PBS- and pcDNA3-treated control groups according to tumor weight. Data shown are representative of three independent experiments. rhIL-24, recombinant human interleukin-24; PBS, phosphate-buffered saline.

cultured and induced by IPTG for $3 \mathrm{~h}$. The rhIL-24 insoluble inclusion body was then collected and denatured. Subsequently, the rhIL-24 soluble protein was renatured and purified through a Q-Sepharose column. SDS-PAGE (Fig. 1A) and western blot analysis (Fig. 1B) revealed that the purified protein was relatively pure rhIL-24 protein. In addition, the rhIL-24 expression levels in the mammalian cells, QBI-293A cells transiently transfected with pcDNA3-hIL-24 and CHO cells stably transfected with pcDNA3-hIL-24, were determined by ELISA analysis of the respective culture supernates (Fig. 1C). To further analyze the stability of the purified bacteria rhIL-24 protein and the naked plasmid-mediated IL-24 expression in vivo, the s.c. xenografted MDA-MB-231 human breast cancer tumors were removed for immunohistochemical analysis of IL-24 at three weeks after the initiation of treatment. In vivo IL-24 expression, following intratumoral injections of soluble rhIL-24 protein, was persistently detected, which indicated that the purified rhIL-24 protein was relatively stable and durable (Fig. 1D). Intratumoral injections of the liposome-coated pcDNA3-hIL-24 naked plasmid were also capable of efficiently inducing in vivo IL-24 transgene expression (Fig. 1D).

rhIL-24 suppresses breast cancer cell growth. To examine the cytotoxic activity of prokarytotic bacteria- and eukaryotic cell-derived rhIL-24 against MDA-MB-231 human breast cancer cells, the cells were treated with purified rhIL-24 protein from prokarytotic BL21 bacteria and rhIL-24 supernate from the CHO eukaryotic mammalian 
A

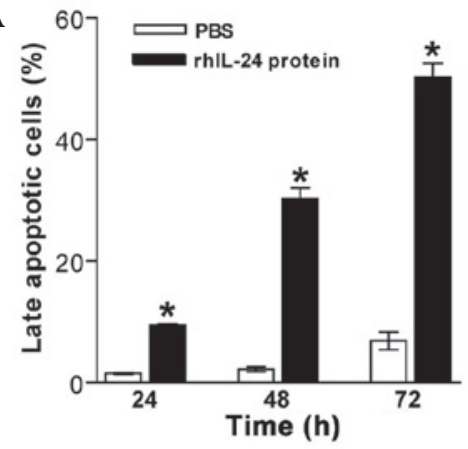

B

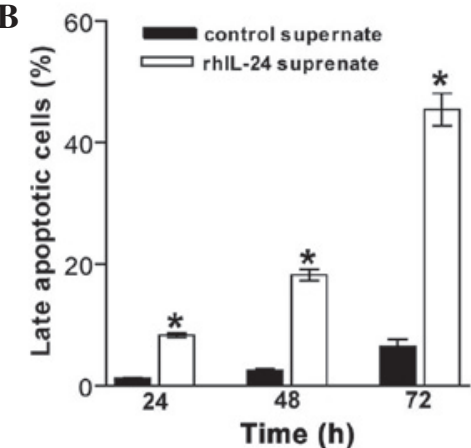

C

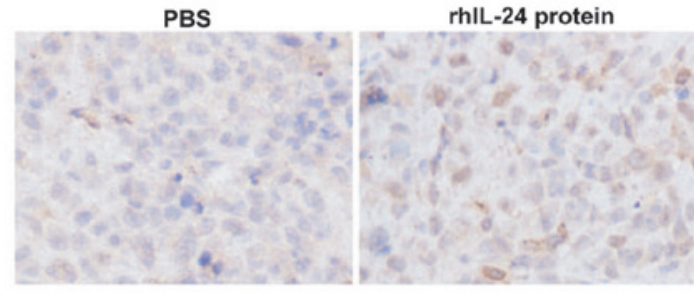

pcDNA3

pcDNA3-hIL-24

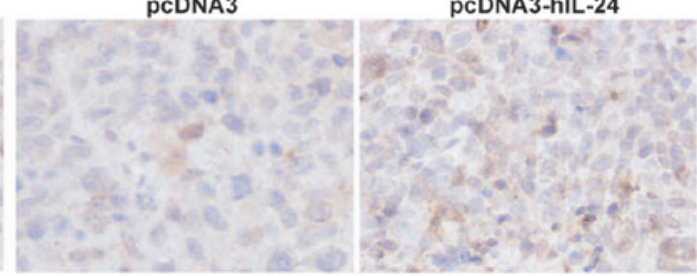

D

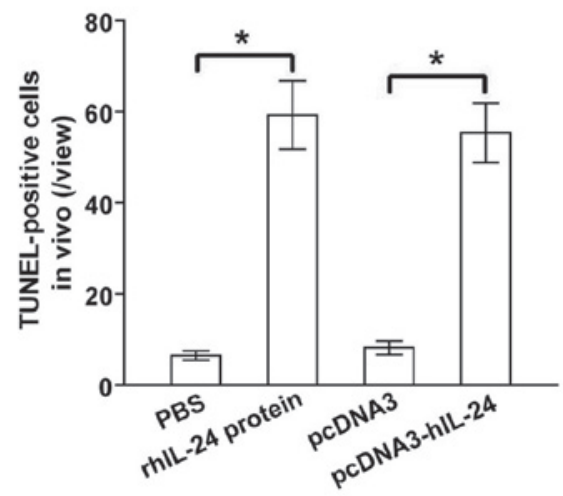

Figure 3. rhIL-24 induces tumor apoptosis in vitro and in vivo. (A and B) Flow cytometric analysis of in vitro apoptosis. The MDA-MB-231 human breast cancer cells were treated with PBS, rhIL-24 protein, control supernate or rhIL-24 supernate. The tumors were harvested at different time points (24-72 h) after treatment, stained with Annexin V-fluorescein isothiocyanate and PI, and then analyzed by flow cytometry. The double Annexin V- and PI-positive cells in the total cell population indicated late apoptotic cells. (A) Apoptotic rate in PBS- and rhIL-24-treated cells. *P<0.05 compared with the PBS-treated cells. (B) Apoptotic rate in cells treated with rhIL-24 or control supernate. ${ }^{*} \mathrm{P}<0.05$ compared with the cells treated with the control supernate. Data were analyzed by the Student's t-test; $n=3$ replicates per condition. (C and D) TUNEL analysis of in vivo apoptosis in MDA-MB-231 subcutaneously xenografted tumors. (C) Representative images of the TUNEL assay. (D) TUNEL-positive cells signified in vivo apoptotic cells. "P<0.05 compared with the PBS and pcDNA3 cells, respectively. $\mathrm{N}=8$, replicates per condition; $\mathrm{n}=5$, sections per replicate; $\mathrm{n}=5$, observations per section. Data are representative of three independent experiments rhIL-24, recombinant human interleukin-24; PBS, phosphate-buffered saline; PI, propidium iodide; TUNEL, terminal deoxynucleotidyl transferase-mediated dUTP nick end labeling.

cells, respectively. Tumor cell viability was assessed daily for five days using an MTT assay. The bacterial rhIL-24 protein and eukaryotically expressed rhIL-24 supernate significantly suppressed in vitro MDA-MB-231 tumor cell growth in a time-dependent manner ( $\mathrm{p}<0.05$; Fig. 2A-C). Furthermore, the in vivo growth of s.c. xenografted MDA-MB-231 cell tumors in athymic nude mice was efficiently retarded when the tumors were treated with rhIL-24 protein or rhIL-24 supernate $(\mathrm{p}<0.05$; Fig. $2 \mathrm{D}-\mathrm{G})$. These results indicated that prokarytotic system-derived renatured rhIL-24 protein and eukaryotic system-derived rhIL-24 were capable of significantly inhibiting MDA-MB-231 tumor growth in vitro and in vivo.

rhIL-24 induces breast cancer apoptosis. To analyze the underlying mechanism of rhIL-24-elicted MDA-MB-231 tumor cell cytotoxicity, the MDA-MB-231 human breast cancer cells were treated with purified rhIL-24 protein or rhIL-24 supernate for 24-72 h. The tumor cells were then harvested and subjected to apoptotic analysis using Annexin V-FITC/PI double-staining. Treatment with rhIL-24 protein induced MDA-MB-231 tumor cell apoptosis in a time-dependent manner (Fig. 3A). As compared with the PBS control group, significantly greater numbers [9.5\% (24 h), 30.3\% (48 h) and 50.2\% (72 h)] of late apoptotic cells were observed in the rhIL-24 protein-treated group $(\mathrm{p}<0.05$; Fig. 3A). Comparable with the apoptosis-inducing effect of the bacterial rhIL-24 protein, the eukaryotically expressed rhIL-24 supernate also significantly induced MDA-MB-231 tumor cell apoptosis $(\mathrm{P}<0.05$; Fig. 3B), in particular at $72 \mathrm{~h}$. To further verify the in vivo induction of apoptosis in rhIL-24 protein- and rhIL-24 supernate-treated, and untreated control 
A

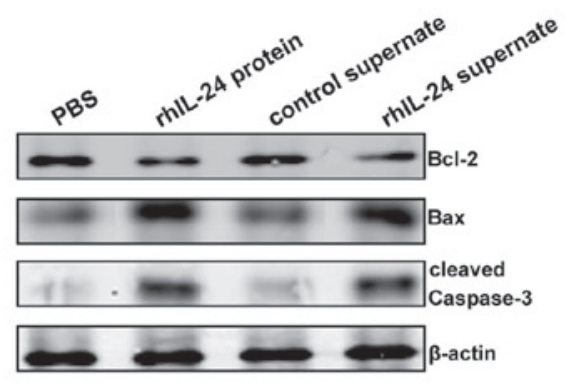

B

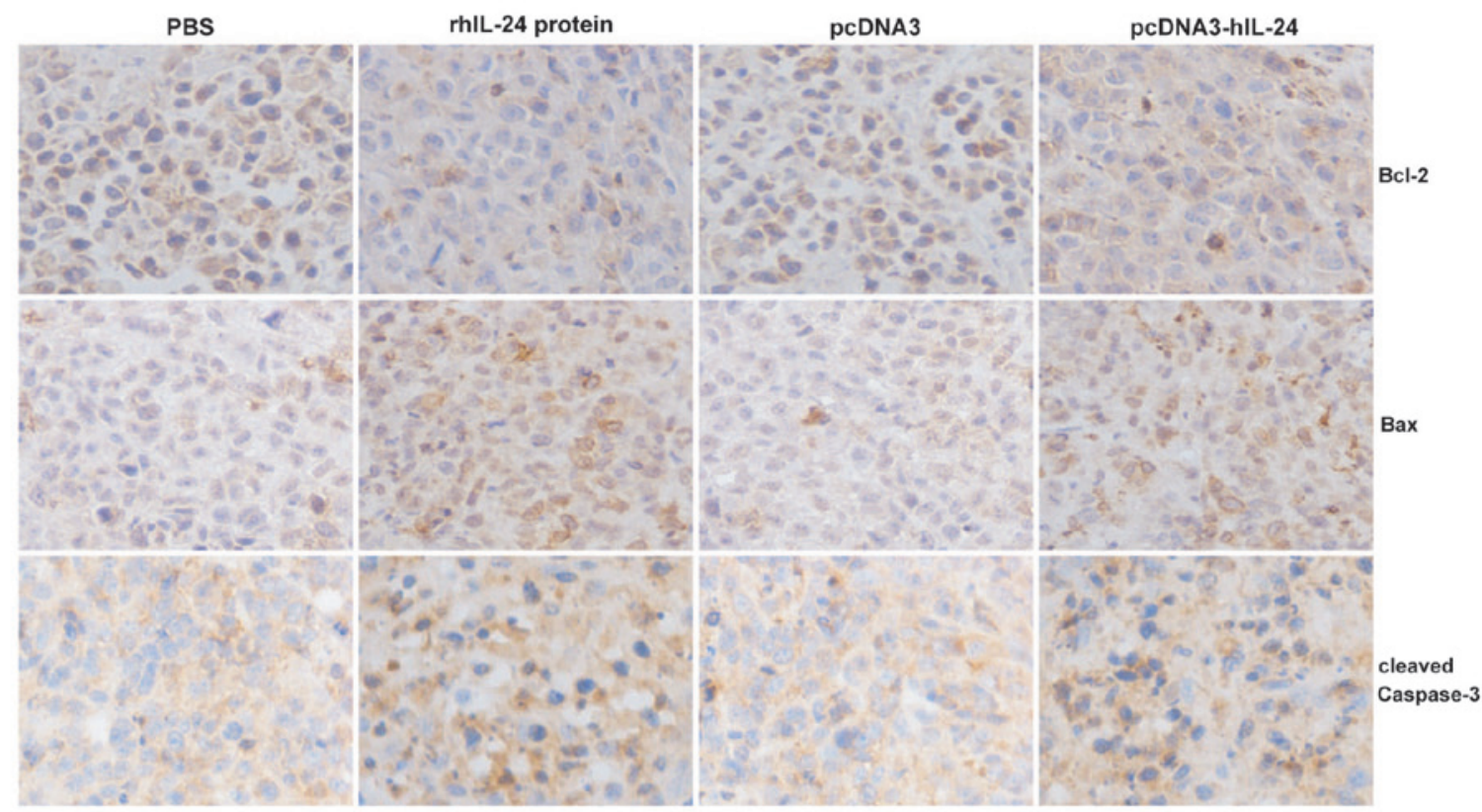

C

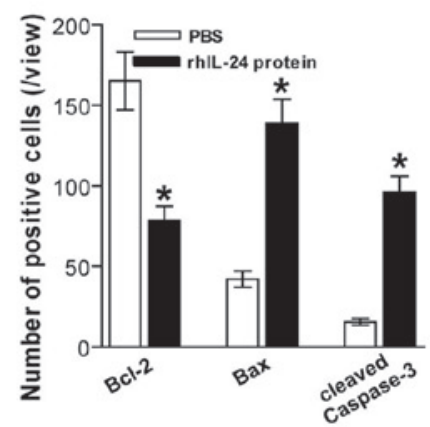

D

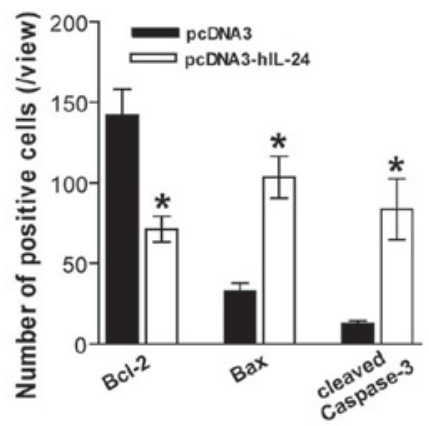

Figure 4. rhIL-24 activates intrinsic apoptosis through upregulation of Bax/Bcl-2. (A) Western blot analysis of apoptosis-associated proteins. The total cellular lysates derived from PBS-, rhIL-24 protein-, control supernate- or rhIL-24 supernate-treated MDA-MB-231 human breast cancer cells were immunoblotted with a panel of antibodies specific for Bcl-2, Bax, cleaved caspase-3 and $\beta$-actin (serving as an internal control), respectively. Representative images from western blot analysis are shown. (B) Representative images of the immunohistochemical detection of Bcl-2, Bax and cleaved caspase-3 in subcutaneously xenografted MDA-MB-231 human breast cancer cell tumors. (C and D) The number of positive cells as determined by immunohistochemical detection. ${ }^{*} \mathrm{P}<0.05$ compared with the respective control conditions. Data were analyzed by the Student's t-test; $\mathrm{n}=8$, replicates per condition; $\mathrm{n}=5$, sections per replicate; $\mathrm{n}=5$, observations per section. Data are representative of three independent experiments. rhIL-24, recombinant human interleukin-24; PBS, phosphate-buffered saline; Bcl-2, B cell lymphoma 2; Bax, Bcl-2-associated X protein.

s.c. xenografted MDA-MB-231 human breast cancer tumors, immunohistochemical analysis (Fig. 3C) and a TUNEL assay (Fig. 3D) were performed. Consistent with the in vitro flow cytometric analysis of apoptosis, the two forms of rhIL-24 protein also significantly induced in vivo apoptosis in MDA-MB-231 tumor cells s.c. implanted in athymic nude mice $(\mathrm{p}<0.05)$.
rhIL-24 upregulates Bax/Bcl-2 and activates caspase-3. To elucidate the underlying molecular mechanism of the IL-24-mediated antitumor effects, the expression levels of Bcl-2 family proteins associated with apoptosis, such as Bcl-2 and Bax, and cleaved caspase-3, in the s.c. xenografted MDA-MB-231 human breast cancer cell tumors following the different treatments were assessed by immunohisto- 
A

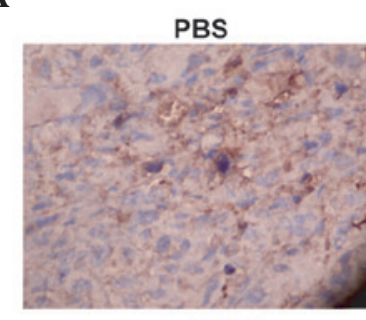

pcDNA3

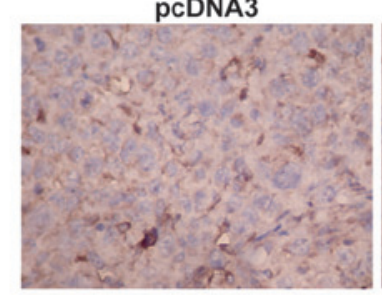

rhIL-24 protein

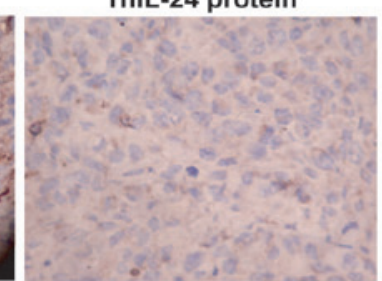

pcDNA3-hIL-24

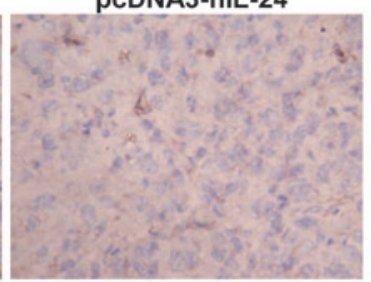

B

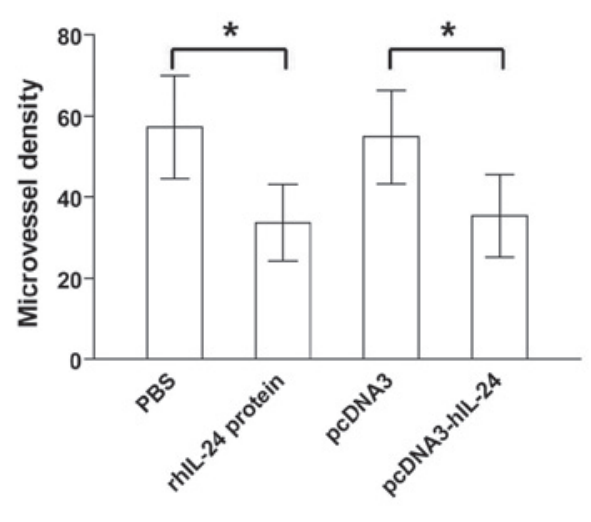

Figure 5. rhIL-24 suppresses tumor angiogenesis in vivo. (A) Representative images of immunohistochemical detection of cluster of differentiation 34 in s.c. xenografted MDA-MB-231 human breast cancer cell tumors. (B) The microvessel density in MDA-MB-231 s.c. xenografted tumors. ${ }^{*}<0.05$. Data were analyzed by the Student's t-test; $n=8$, replicates per condition; $n=5$, sections per replicate; $n=5$, observations per section. Data are representative of three independent experiments. rhIL-24, recombinant human interleukin-24; PBS, phosphate-buffered saline; s.c., subcutaneously.

chemistry. The expression levels of the Bcl-2 antiapoptotic molecule in the rhIL-24 protein and rhIL-24 supernate groups were significantly reduced, whereas the proapoptotic molecule Bax expression levels in these groups were significantly increased (Fig. 4A). Significantly increased cleaved caspase-3 expression levels were also observed in the rhIL-24 protein and rhIL-24 supernate groups (Fig. 4A). These results indicated that bacteria rhIL-24 protein and eukaryotically expressed rhIL-24 supernate treatment efficiently induced MDA-MB-231 breast cancer apoptosis via upregulation of the $\mathrm{Bax} / \mathrm{Bcl}-2$ ratio and activation of caspase-3. The effect of the rhIL-24 protein and the pcDNA3-hIL-24 naked plasmid expressing human IL-24 on the in vivo expression levels of Bcl-2, Bax and cleaved caspase-3 in s.c. xenografted MDA-MB-231 cell tumors was further verified by immunohistochemical analysis (Fig. 4B-D).

rhIL-24 inhibits tumor angiogenesis. Positive CD34 expression was predominantly evident as brownish yellow or brownish granules in the vascular endothelial cells of the s.c. xenografted MDA-MB-231 human breast cancer cell tumors (Fig. 5A). As compared with the respective PBS and pcDNA3 control groups, the CD34 expression levels in tumor vascular endothelial cells in the rhIL-24 protein and pcDNA3-hIL-24 treated groups were reduced (Fig. 5A). Furthermore, the MVD values (Fig. 5B) counted in the rhIL-24 protein and pcDNA3-hIL-24 groups were significantly less than those in the respective PBS and pcDNA3 control groups $(\mathrm{p}<0.05)$. These data indicated that treatment with the bacterial rhIL-24 protein and the pcDNA3-hIL-24 naked plasmid expressing human IL-24 efficiently suppressed in vivo tumor angiogenesis in xenografted MDA-MB-231 breast cancer cell tumors.

\section{Discussion}

Numerous studies have revealed that cytokine-tumor suppressor IL-24 inhibits tumor cell growth and induces apoptosis in a large variety of cancer cell types via the acti- vation of double-stranded RNA-dependent protein kinase, p38 mitogen-activated protein kinase, c-Jun $\mathrm{NH}_{2}$-terminal kinase and endoplasmic reticulum stress-mediated unfolded protein response signaling pathways, and the inhibition of the $\beta$-catenin and PI3K signaling pathways $(3,19)$. Furthermore, IL-24 as a cytokine may be processed via class secretory pathways, bind specific cytokine receptor complexes (IL-20R1/IL-20R2 and IL-20R2/IL-22R), and consequently activate the Janus kinase/Signal Transducer and Activator of Transcription signaling pathway $(3,19)$. The ability of IL-24 to discriminate between normal and tumor cells, induce apoptosis, suppress tumor angiogenesis, stimulate immune responses, promote bystander antitumor activity, and synergize with anticancer drugs and radiation render IL-24 a strong tumor suppressor in cancer treatment. Adenovirus-mediated IL-24 gene therapy (Ad.mda-7 and INGN 241) is currently undergoing phase I clinical trials for the treatment of solid tumors $(19,20)$. Bacterially expressed rhIL-24 protein has also been demonstrated to exert cancer-specific killing activity, providing an alternate therapeutic reagent for cancer therapy (16).

rhIL-24 protein has been previously demonstrated to exert in vitro and in vivo antitumor effects in lung cancer (21) and gastric cancer (22). In the present study, the antitumor activity of bacteria- and CHO mammalian cell-derived rhIL-24 protein, and pcDNA3-hIL-24 naked plasmid were assessed in the IL-20R1/IL-20R2-expressing MDA-MB-231 human breast cancer cell line in vitro and in vivo. The data revealed that the prokarytotic system-derived rhIL-24 protein and the eukaryotic system-derived secretory rhIL-24 protein were capable of efficiently suppressing MDA-MB-231 tumor growth in vitro. In the animal experiments, the bacterially expressed rhIL-24 protein and the pcDNA3-hIL-24 naked plasmid exerted significant antitumor activity on subcutaneously xenografted MDA-MB-231 breast cancer cell tumors. Flow cytometric analysis and TUNEL assay further verified that the antitumor efficacy of rhIL-24 in breast cancer was dependent on the induction of apoptosis. These results were consistent with 
those of a previous study that observed that N-glycosylation of IL-24 was not mandatory for tumor cell-specific apoptosis or bystander antitumor activity (23).

The ratio of Bax/Bcl-2 pro- to antiapoptotic molecules constitutes a rheostat that sets the threshold of susceptibility to caspase-3 activation and apoptosis for the intrinsic pathway (24). In the present study, to elucidate the underlying molecular mechanism responsible for the induction of apoptosis, the Bcl-2 and Bax apoptosis-associated proteins and cleaved caspase- 3 were examined using western blot analysis and immunohistochemistry. Therefore, the rhIL-24-elicited upregulation of Bax/Bcl-2 and the activation of caspase-3 may contribute to IL-24-induced tumor suppression in breast cancer.

Tumor angiogenesis is also a prerequisite for successful tumor growth and formation of metastases (25). Antiangiogenic therapy is regarded as a potential and nontoxic therapeutic strategy in cancer therapy (26). A great deal of data have revealed that IL-24 inhibits tumor angiogenesis via direct interaction with IL-20R2/IL-22R on vascular endothelial cells (4) and via an indirect reduction in the production of proangiogenic factor $(5,6)$. In the present study, CD34 immunohistochemical analysis revealed that rhIL-24 downregulated CD34 expression and reduced MVD in s.c. xenografted breast cancer cell tumors, which may be an additional important mechanism responsible for rhIL-24-mediated in vivo breast cancer growth suppression in athymic nude mice.

In conclusion, in the present study, prokaryotically and eukaryotically expressed rhIL-24 were demonstrated to efficiently suppress MDA-MB-231 tumor growth in vitro. Similarly, bacteria-derived rhIL-24 protein and pcDNA3-hIL-24 naked plasmid administration also provided therapeutic benefits for xenografted MDA-MB-231 cell tumors in vivo. The retarded breast cancer growth elicited by rhIL-24 was closely associated with the induction of apoptosis induced by upregulation of the Bax/Bcl-2 ratio and activation of caspase-3, and the marked inhibition of tumor angiogenesis. Thus, these results indicate that prokaryotically expressed rhIL-24 protein may be an alternate and promising antitumor agent for the treatment of human breast cancer or other types of cancer.

\section{Acknowledgements}

This study was supported by the National Natural Science Foundation of China (grant no. 81001016).

\section{References}

1. Sauane M, Gopalkrishnan RV, Sarkar D, Su ZZ, Lebedeva IV, Dent P, et al: MDA-7/IL-24: novel cancer growth suppressing and apoptosis inducing cytokine. Cytokine Growth Factor Rev 14: 35-51, 2003.

2. Fisher PB: Is mda-7/IL-24 a 'magic bullet' for cancer? Cancer Res 65: 10128-10138, 2005.

3. Whitaker EL, Filippov VA and Duerksen-Hughes PJ: Interleukin 24: Mechanisms and therapeutic potential of an anti-cancer gene. Cytokine Growth Factor Rev 23: 323-331, 2012.

4. Ramesh R, Mhashilkar AM, Tanaka F, Saito Y, Branch CD, Sieger K, et al: Melanoma differentiation-associated gene 7/interleukin (IL)-24 is a novel ligand that regulates angiogenesis via the IL-22 receptor. Cancer Res 63: 5105-5113, 2003.

5. Saeki T, Mhashilkar A, Swanson X, Zou-Yang XH, Sieger K, Kawabe $\mathrm{S}$, et al: Inhibition of human lung cancer growth following adenovirus-mediated mda-7 gene expression in vivo Oncogene 21: 4558-4566, 2002.
6. Nishikawa T, Ramesh R, Munshi A, Chada S and Meyn RE: Adenovirus-mediated mda-7 (IL24) gene therapy suppresses angiogenesis and sensitizes NSCLC xenograft tumors to radiation. Mol Ther 9: 818-828, 2004.

7. Caudell EG, Mumm JB, Poindexter N, Ekmekcioglu S, Mhashilkar AM, Yang XH, et al: The protein product of the tumor suppressor gene, melanoma differentiation-associated gene 7, exhibits immunostimulatory activity and is designated IL-24. J Immunol 168: 6041-6046, 2002.

8. Ramesh R, Ito I, Gopalan B, Saito Y, Mhashilkar AM and Chada S: Ectopic production of MDA-7/IL-24 inhibits invasion and migration of human lung cancer cells. Mol Ther 9: 510-518, 2004.

9. Sauane M, Su ZZ, Gupta P, Lebedeva IV, Dent P, Sarkar D and Fisher PB: Autocrine regulation of mda-7/IL-24 mediates cancer-specific apoptosis. Proc Natl Acad Sci USA 105: 9763-9768, 2008.

10. Hamed HA, Das SK, Sokhi UK, Park MA, Cruickshanks N, Archer K, et al: Combining histone deacetylase inhibitors with MDA-7/IL-24 enhances killing of renal carcinoma cells. Cancer Biol Ther 14: 1039-1049, 2013.

11. Jemal A, Bray F, Center MM, Ferlay J, Ward E and Forman D: Global cancer statistics. CA Cancer J Clin 61: 69-90, 2011.

12. Tinoco G, Warsch S, Glück S, Avancha K and Montero AJ Treating breast cancer in the 21st century: Emerging biological therapies. J Cancer 4: 117-132, 2013.

13. Patani N, Douglas-Jones A, Mansel R, Jiang W and Mokbel K: Tumour suppressor function of MDA-7/IL-24 in human breast cancer. Cancer Cell Int 10: 29, 2010.

14. Frewer NC, Ye L, Sun PH, Owen S, Ji K, Frewer KA, et al: Potential implication of IL-24 in lymphangiogenesis of human breast cancer. Int J Mol Med 31: 1097-1104, 2013.

15. Sarkar D, Su ZZ, Vozhilla N, Park ES, Gupta P and Fisher PB: Dual cancer-specific targeting strategy cures primary and distant breast carcinomas in nude mice. Proc Natl Acad Sci USA 102: 14034-14039, 2005.

16. Sauane M, Gopalkrishnan RV, Choo HT, Gupta P, Lebedeva IV, Yacoub A, et al: Mechanistic aspects of mda-7/IL-24 cancer cell selectivity analysed via a bacterial fusion protein. Oncogene 23: 7679-7690, 2004

17. Pei DS, Yang ZX, Zhang BF, Yin XX, Li LT, Li HZ and Zheng JN: Enhanced apoptosis-inducing function of MDA-7/IL-24 RGD mutant via the increased adhesion to tumor cells. J Interferon Cytokine Res 32: 66-73, 2012.

18. Weidner N: Current pathologic methods for measuring intratumoral microvessel density within breast carcinoma and other solid tumors. Breast Cancer Res Treat 36: 169-180, 1995.

19. Lebedeva IV, Emdad L, Su ZZ, Gupta P, Sauane M, Sarkar D, et al: mda-7/IL-24, novel anticancer cytokine: Focus on bystander antitumor, radiosensitization and antiangiogenic properties and overview of the phase I clinical experience (Review). Int J Oncol 31: 985-1007, 2007.

20. Cunningham CC, Chada S, Merritt JA, Tong A, Senzer N, Zhang Y, et al: Clinical and local biological effects of an intratumoral injection of mda-7 (IL24; INGN 241) in patients with advanced carcinoma: a phase I study. Mol Ther 11: 149-159, 2005.

21. Xie Y, Sheng W, Xiang J, Ye Z, Zhu Y, Chen X and Yang J: Recombinant human IL-24 suppresses lung carcinoma cell growth via induction of cell apoptosis and inhibition of tumor angiogenesis. Cancer Biother Radiopharm 23: 310-320, 2008.

22. Yan S,Zhang H, Xie Y, Sheng W, Xiang J, YeZ, et al: Recombinant human interleukin-24 suppresses gastric carcinoma cell growth in vitro and in vivo. Cancer Invest 28: 85-93, 2010.

23. Sauane M, Gupta P, Lebedeva IV, Su ZZ, Sarkar D, Randolph A, et al: N-glycosylation of MDA-7/IL-24 is dispensable for tumor cell-specific apoptosis and 'bystander' antitumor activity. Cancer Res 66: 11869-11877, 2006.

24. Danial NN and Korsmeyer SJ: Cell death: Critical control points. Cell 116: 205-219, 2004.

25. Hanahan D and Weinberg RA: Hallmarks of cancer: The next generation. Cell 144: 646-674, 2011.

26. Welti J, Loges S, Dimmeler S and Carmeliet P: Recent molecular discoveries in angiogenesis and antiangiogenic therapies in cancer. J Clin Invest 123: 3190-3200, 2013. 